\author{
Francesco G. De Rosa \\ Jason A. Roberts
}

\section{Amikacin dosing in the ICU: we now know more, but still not enough...}

Received: 10 April 2014

Accepted: 15 April 2014

Published online: 10 May 2014

(C) Springer-Verlag Berlin Heidelberg and ESICM 2014

F. G. De Rosa

Department of Medical Sciences, University of Turin, Turin, Italy

F. G. De Rosa

Infectious Diseases at Amedeo di Savoia Hospital, Corso Svizzera 164, 10149 Turin, Italy

\section{J. A. Roberts (}

Burns Trauma and Critical Care Research Centre, Level 3 Ned Hanlon Building, Royal Brisbane and Women's Hospital, The University of Queensland, Butterfield St, Brisbane, Queensland 4029, Australia

e-mail: j.roberts2@uq.edu.au

Tel.: +61-7-36464108

\section{J. A. Roberts}

Royal Brisbane and Women's Hospital, Brisbane, Australia

J. A. Roberts

Department of Molecular and Clinical Pharmacology, The

University of Liverpool, Liverpool, UK of concentration targets in a heterogeneous patient cohort. They found that even with a $25 \mathrm{mg} / \mathrm{kg}$ loading dose, $33 \%$ of patients still did not achieve Cmax/MIC ratio [ratio of maximum concentration during dosing interval (Cmax) to the minimum inhibitory concentration of the known/suspected pathogen (MIC)] of $8-10$, assuming the highest MIC of susceptible pathogens.

\section{Why is this not a perfect study?}

Firstly, the weight metric selected by the authors was total body weight and not an adjusted dosing weight, which is considered more appropriate, particularly for obese patients. Secondly, there are patients with acute kidney injury as well as patients treated with continuous renal replacement therapy which should require a slightly reduced loading dose compared with patients with better renal function. Thirdly, there was an insufficient patient enrolment to determine microbiological outcomes associated with altered concentrations. Finally, no analyses were provided to show how a clinician can overcome the inadequacies of a fixed dosing approach or even what dose should be used. Here a population pharmacokinetic analysis with dosing simulations would be preferred, but is unfortunately not possible with the current study design.

\section{Why is this a useful study?} as the paper by de Montmollin et al. [1] in this issue of Intensive Care Medicine, which uses a simple study design to provide a strong and clinically relevant message that is very useful for daily work in the intensive care unit (ICU). Although a standard dosage of $15 \mathrm{mg} / \mathrm{kg}$ once daily is commonly used for amikacin, the authors evaluated the effect of a higher fixed weight-based amikacin dose in critically ill patients, $25 \mathrm{mg} / \mathrm{kg}$, on achievement
Antibiotic pharmacokinetics is clearly different in ICU patients compared with ward-based patients [2]. By including a large heterogenous casemix of patients including those with different levels of renal function, different levels of fluid balance and different pathologies such as ascites and cirrhosis, the authors were able 


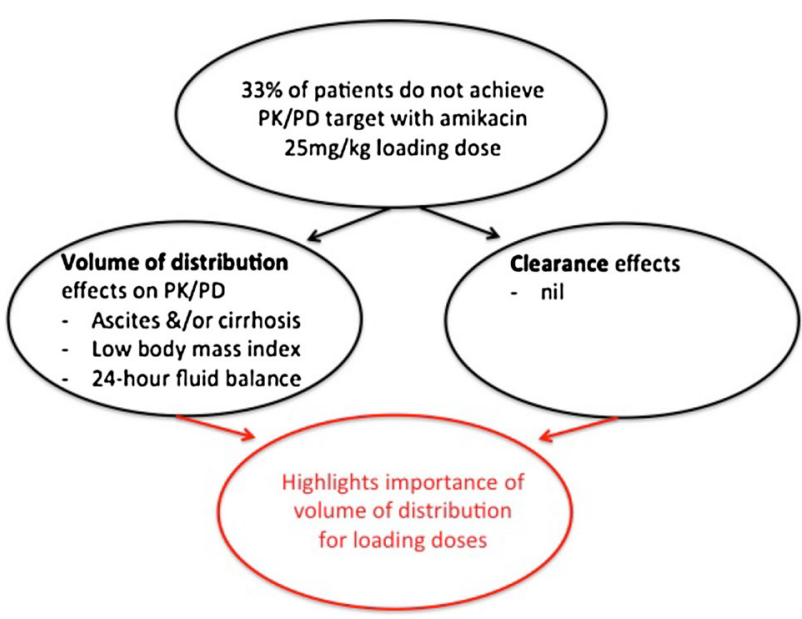

Fig. 1 Patient and treatment factors that affect amikacin volume of distribution rather than clearance and resulted in sub-optimal achievement of target concentration

to statistically evaluate which factors were associated with suboptimal achievement of concentration targets. In fact the authors should be complimented for the large number of patients recruited. In the context of other pharmacokinetic studies in the ICU, this is a very large patient sample. The results of univariate analysis showed that the presence of ascites, cirrhosis and an increasing 24-h fluid intake or 24-h fluid balance were significantly related to lower Cmax. Each of these factors logically would increase amikacin volume of distribution which would lead to a lower Cmax. Interestingly, the multivariate analysis showed that only a body mass index (BMI) less than $25 \mathrm{~kg} / \mathrm{m}^{2}$ and the 24-h fluid balance were significantly associated with a lower Cmax. As shown in Fig. 1, the data from this study is useful as it highlights the importance of factors that affect amikacin volume of distribution on the success of loading doses and that factors related to clearance are not relevant (although this may be because the authors did not choose a maximum accepted amikacin concentration).

\section{Why is this study important, controversial and thought-provoking?}

Given that BMI and not weight was shown to be a predictor of low amikacin concentrations, should we be dosing based on BMI? Drug dosing based on BMI has been proposed previously but is generally considered to be less accurate than other weight metrics like lean body weight or fat free mass [3]. The authors discuss why they used total body weight and not other weight metrics in this cohort that has few obese patients. However, in other countries there is no way that total body weight could be used because of the prevalence of obesity. Best evidence currently supports use of adjusted body weight in obese patients as highlighted by the authors in the discussion. Ideally a study design using lean or adjusted body weight would be appropriate for a hydrophilic drug like amikacin, but determining lean body weight in critically ill patients is technically difficult and so adjusted body weight is appropriate [4].

Since this is not the perfect paper but does target a basic question, it leaves us with some doubts: how many patients are treated with lower dose aminoglycosides, like amikacin, in other ICUs and how frequently are these patients not achieving target aminoglycoside concentrations? How many of these patients are given aminoglycoside doses which may increase the likelihood of generating and amplifying antibiotic resistance? The big question is in the conclusion: how much higher should empiric aminoglycoside doses be and which patients should receive them? These would be clinical messages that would further enhance our understanding of this area, although de Montmollin and colleagues have provided important data to help direct further research in this area. In the context of similar recent data from other antibiotics $[5,6]$, this problem should not be considered restricted to aminoglycosides. Indeed, quantification of the clinical advantages of an individualized approach to dosing is urgently required.

Acknowledgments Professor Roberts is funded by a Career Development Fellowship from the National Health and Medical Research Council of Australia (APP1048652).

Conflicts of interest Neither author has any conflicts to declare.

\section{References}

1. de Montmollin E, Bouadma L, Gault N, 2. Roberts JA (2011) Using PK/PD to Mourvillier B, Mariotte E, Chemam S, Massias L, Papy E, Tubach F, Wolff M, Sonneville R (2014) Predictors of insufficient amikacin peak concentration in critically ill patients receiving a $25 \mathrm{mg} / \mathrm{kg}$ total body weight regimen. Intensive Care Med. doi: $10.1007 / \mathrm{s} 00134-014-3276-\mathrm{x}$ ill patients. Curr Pharm Biotechnol 12:2070-2079
3. Janmahasatian S, Duffull SB, Ash S, Ward LC, Byrne NM, Green B (2005) Quantification of lean bodyweight. Clin Pharmacokinet 44:1051-1065 
4. Roberts JA, Roberts MS, Semark A, Udy AA, Kirkpatrick CM, Paterson DL, Roberts MJ, Kruger P, Lipman J (2011) Antibiotic dosing in the 'at risk' critically ill patient: linking pathophysiology with pharmacokinetics/ pharmacodynamics in sepsis and trauma patients. BMC Anesthesiol 11:3
5. Roberts JA, Paul SK, Akova M, Bassetti M, De Waele JJ, Dimopoulos G, Kaukonen KM, Koulenti D, Martin C, Montravers P, Rello J, Rhodes A, Starr T, Wallis SC, Lipman J, Study D (2014) DALI: defining antibiotic levels in intensive care unit patients: are current beta-lactam antibiotic doses sufficient for critically ill patients? Clin Infect Dis 58:1072-1083
6. Roberts JA, Stove V, De Waele JJ, Sipinkoski B, McWhinney B, Ungerer JP, Akova M, Bassetti M, Dimopoulos G, Kaukonen KM, Koulenti D, Martin C, Montravers P, Rello J, Rhodes A, Starr T, Wallis SC, Lipman J, on behalf of the DALI Study authors (2014) Variability in protein binding of teicoplanin and achievement of therapeutic drug monitoring targets in critically ill patients: lessons from the DALI Study. Int J Antimicrob Agents 43(5):423-430 